\title{
Multithreaded Conductive Carbon: 1D Conduction in 3D Carbon
}

Yilong Pan, Meng Hu, Mengdong Ma, Zihe Li, Yufei Gao, Mei Xiong, Guoying Gao, Zhisheng Zhao, Yongjun Tian, Bo Xu, Julong He

\begin{abstract}
Carbon possesses various allotropes with diverse electronic properties from insulation to conduction due to the $s p, s p^{2}$, and $s p^{3}$ bonding types. Here, a new $s p^{2}-s p^{3}$ carbon allotrope, called $\mathrm{C}_{48}$, is proposed. $\mathrm{C}_{48}$ presents a lilac-like structure with a cage-like center and four petal-like tubes around, and can be formed through compressing single-walled carbon nanotubes. $\mathrm{C}_{48}$ is not only strong and hard in $3 \mathrm{D}$ but also has unexpected 1D conduction along eight divided linear pathways in the unit cell. This multithreaded 1D conduction in 3D framework is peculiar in carbon materials, and would be helpful to design singular electronic nanodevices.
\end{abstract}




\section{Introduction}

Elemental carbon fosters various polymorphs, such as graphite, diamond, graphene, and carbon nanotubes (CNTs), due to the ability to form $s p, s p^{2}$, and $s p^{3}$ bonds. The carbon allotropes with different bond types reveal distinct electronic properties. Generally, all- $s p^{3}$ carbons including diamond and theoretical M-carbon[1], bct- $\mathrm{C}_{4}[2,3]$, W-carbon[4], and $\mathrm{Cco}_{-} \mathrm{C}_{8}[5]$ are semiconductors because all valence electrons are tightly localized in the covalent bonds. As an exception, Tri-carbon with bent $s p^{3}$ bonds shows metallicity contributed to the extremely anisotropic bonds featuring $\pi$ state characteristics[6]. On the other hand, most of the theoretical metallic carbons, such as K4-carbon[7], T6-carbon, T14-carbon[8], and some 3D CNT polymers[9], are all-sp ${ }^{2}$ or $s p^{2}-s p^{3}$ hybridized with conductivity originated from the delocalized electrons of $s p^{2}$ atoms similar to that of graphite, graphene and CNTs. By manipulating the location of $s p^{2}$ and $s p^{3}$ atoms, the 3D carbons with controllable conduction can be designed expectedly, and the deeper thinking about their formation need to be worthy of attention.

As is well-known, pressure-induced changes of bonding from $s p^{2}$ to $s p^{3}$ for $s p^{2}$-hybridized carbons such as graphite, fullerenes, and CNTs are the key for producing new carbon phases with novel electronic electrical properties and superior mechanical performance. Due to the difference of crystal structures and hybridized forms of raw carbons, these carbon precursors would undergo distinct phase transitions under pressure due to the kinetic factor. Recently, transition behavior of zigzag $(n, 0)$ and armchair $(n, n)$ single-walled carbon nanotubes (SWCNTs) under hydrostatic and 
nonhydrostatic pressures were systematically studied. Some new carbons were revealed such as 3D (2, 2)-II (Bct-C 4 [10]), 3D (2, 2)-III (Cco-C $\left.{ }_{8}[5]\right), 3 \mathrm{D}(3,3)-\mathrm{I}[11]$, 3D (5, 0)-III[12], 3D (6, 0)-I[13], 3D (7, 0)[12], and 3D $(8,8)$ carbon[13]. Among them, all-sp $p^{3}$ carbons would be readily formed by compressing SWCNTs with relatively small diameter diameters[5]. Meanwhile, mixed $s p^{2}-s p^{3}$ hybridized carbons were usually obtained from compressing large-diameter SWCNTs[12, 13]. Considering these $s p^{2}-s p^{3}$ carbons commonly possess the 3D conductivity, it is highly desirable to search new 3D carbons displaying peculiar 1D or 2D electronic properties, which would be attractive to be applied in specific-specified conduction of nano-electronic devices.

In this study, a $s p^{2}-s p^{3} 3 \mathrm{D}$ carbon, called $\mathrm{C}_{48}$, with novel 1D multithreaded eonductivity conduction is proposed. The $\mathrm{C}_{48}$ has a charming lilac-like structure with a cage-like center composed of $s p^{3}$ bonds as well as four surrounding surrounded petal-like tubes of both $s p^{2}$ and $s p^{3}$ hybridizations. The $s p^{2}$ atoms are specifically located at the divided area, endowing the multithreaded linear conductivity conduction. Due to the intervention of $s p^{3}$ atoms, the mechanical properties such as strength, hardness, and modulus have been improved in comparison with that those of pure $s p^{2}$ carbons. Furthermore, the $\mathrm{C}_{48}$ has also been proved to be formed by direct compression of large-diameter $(12,12)$ SWCNT bundle, demonstrating its viability. The multithreaded $1 \mathrm{D}$ conduction in $3 \mathrm{D}$ carbon framework has the conductive behavior distinct from 3D eonductivity conduction of graphite and 2D eonductivity conduction of Dirac graphene, presenting the great potential of carbon materials. 


\section{Calculation methods}

To obtain the reasonable $s p^{2}-s p^{3}$ carbon configurations, the raw bundles of SWCNTs with tube diameter as large as $1.627 \mathrm{~nm}$ and intertube distance of $0.34 \mathrm{~nm}$ were built through the Materials Studio software, and then they were compressed at different pressures. Structural relaxations and property calculations were performed based on the density functional theory (DFT) as implemented in the CASTEP code[14]. The Vanderbilt ultrasoft pseudopotential was used and the electron-electron exchange interaction was described by the exchange-correlation function of Ceperley and Alder, as parameterized by Perdew and Zunger (CA-PZ) of the local-density approximation (LDA)[15, 16]. The plane-wave cutoff energy was $310 \mathrm{eV}$, and a $k$-point spacing $\left(2 \pi \times 0.04 \AA^{-1}\right)$ was used to generate Monkhorst-Pack $k$-points grids for Brillouin zone sampling[17]. The finite displacement method was used to calculate the phonon frequencies of $\mathrm{C}_{48}$. The unit cells were chosen to calculate the band structures, bulk modulus, shear modulus and elastic constants. The Vickers hardness was estimated using Chen's hardness model[18, 19].

\section{Results and discussion}

The designed $\mathrm{C}_{48}$ has a tetragonal structure (space group: P4/mmm, No.123) with 48 atoms per unit cell. Fig. 1 shows a perspective view of $\mathrm{C}_{48}$ with a cage-like center surrounded by four squashed tubes, and the optimized lattice parameters and Wyckoff atomic positions at ambient pressure are also listed. There are six kinds of nonequivalent carbon atoms in the unit cell including two kinds of $s p^{2}$ gray atoms 
separated by other $s p^{3}$ black atoms. The $s p^{2}$ gray atoms are linearly connected along $z$ axis, but are disconnected in the $x-y$ plane. The bond angle of $s p^{2}$ atoms is not the perfect $120^{\circ}$ but $122.02^{\circ}$ due to the bond bending. The thermodynamic stability of $\mathrm{C}_{48}$ is eompared comparable with those of experimental and theoretical carbon allotropes.

The $\mathrm{C}_{48}$ has an ambient-pressure enthalpy of $0.43 \mathrm{eV} /$ atom higher than that of graphite, and thereby is metastable relative to graphite, diamond, and large-diameter SWCNTs at ambient pressure. However In spite of this, $\mathrm{C}_{48}$ has a similar enthalpy with that of fullerene $\mathrm{C}_{60}$, and is more stable than some theoretically proposed $s p^{2}-s p^{3}$ compressed SWCNT structures like 3D (3, 3)-I[11], 3D (6, 6)-I[13], and 3D $(8,8)[13]$, indicating its viability.

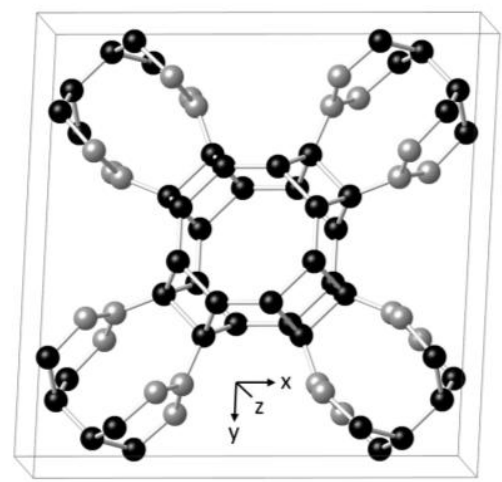

(a)

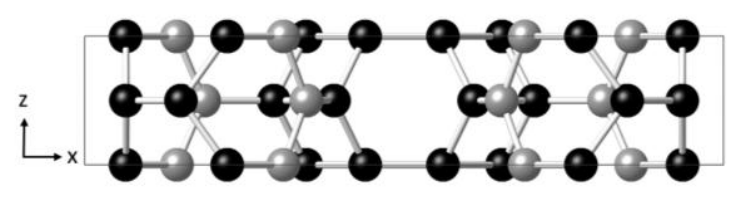

(b)

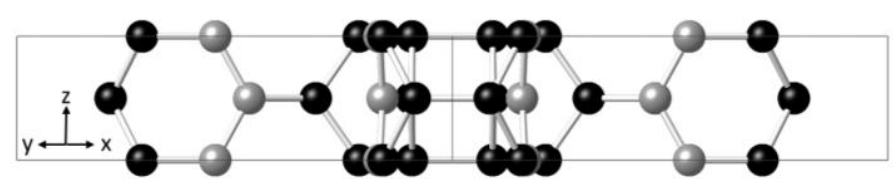

(c)

Figure 1. The crystal structure of $\mathrm{C}_{48}$ (a) and its side views along the [100] (b) and [110] (c) directions, respectively. The $s p^{2}$ and $s p^{3}$ hybridized atoms are colored as gray and black. The $\mathrm{C}_{48}$ has a space group of $P 4 / \mathrm{mmm}$ with ambient-pressure lattice parameters of $a=12.35 \AA, c=2.47$ $\AA$, and Wyckoff atomic positions of $8 q(0.153,0.939,0.5), 8 q(0.356,0.189,0.5), 8 q(0.436$, $0.277,0.5), 8 p(0.204,0.110,0), 8 p(0.435,0.349,0)$, and $8 p(0.313,0.153,0)$.

Guaranteeing structurally the mechanical stability of structure is essential before property predictions. The calculated elastic constants of $\mathrm{C}_{48}$ are $C_{11}=382.15$, $C_{33}=963.16, C_{44}=247.54, C_{66}=141.52, C_{12}=259.68$, and $C_{13}=48.25 \mathrm{GPa}$, respectively, 
which meet the mechanical stability criteria for a tetragonal phase, i.e. $C_{11}>0, C_{33}>0$, $C_{44}>0, C_{66}>0, C_{11}>\mathrm{C}_{12}, C_{11}+C_{33}>2 C_{13},\left(2 C_{11}+2 C_{12}+C_{33}+4 C_{13}\right)>0[20]$. As a result, the $\mathrm{C}_{48}$ is mechanically stable. The bulk (B) and shear (G) moduli of $\mathrm{C}_{48}$ are 264.70 and 182.63 GPa, respectively. The B/G ratio[21] and Poisson's ratio[22] of $\mathrm{C}_{48}$ are 1.45 and 0.22 , respectively. Both of them are higher than that of diamond (0.82 and 0.07) and cubic $\mathrm{BN}(0.97$ and 0.12$)$, indicating it is more ductile than the two superhard materials. The ambient-pressure phonon dispersion curves at ambient pressure was were

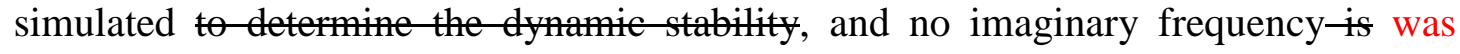
detected throughout the whole Brillouin zone (Fig.2b), demonstrating the dynamic stability of $\mathrm{C}_{48}$. Therefore, the $\mathrm{C}_{48}$ is thermodynamically, mechanically, and dynamically stable, and can be theoretically quenched to ambient pressure.

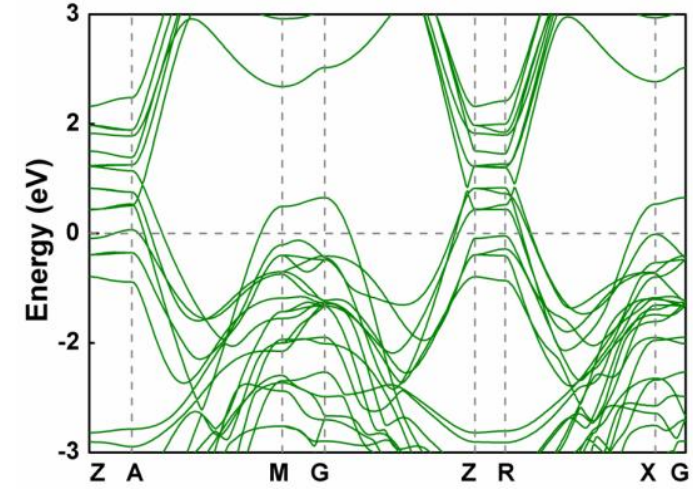

(a)

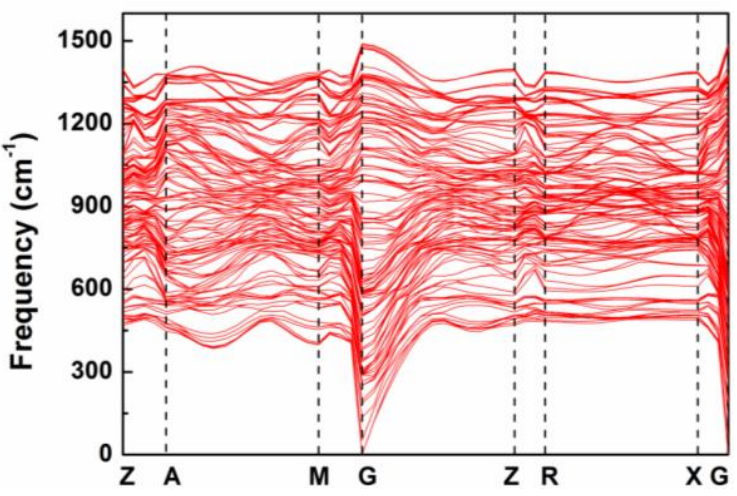

(b)

Figure 2. Ambient-pressure electronic band structure (a) and phonon dispersion curves (b) of $\mathrm{C}_{48}$.

To explore the potential unique electric electrical properties, we calculated the electronic band structure (Fig.2a), and overall it shows that $\mathrm{C}_{48}$ is a conductor. Seen from the band structure, several flat bands crossed $\mathrm{Z}$ and $\mathrm{R}$ points as well as $\mathrm{Z}$ and $\mathrm{A}$ 
points appear at the Fermi level. Meanwhile, some steep conduction bands along $\mathrm{M}$ and $\mathrm{G}$ points toward to $\mathrm{A}$ or $\mathrm{Z}$ point as well as $\mathrm{Z}$ and $\mathrm{R}$ points toward to $\mathrm{G}$ or $\mathrm{X}$ point are observed to pass through the Fermi level repeatedly. The contemporary existence of flat and steep bands at the Fermi level can be regarded as a "fingerprint" for a superconducting compound[23], indicating the possible superconductivity of $\mathrm{C}_{48}$.

In order to figure out the conductive mechanism, we further calculated the electronic partial density of states (PDOS), and electron localization functions (ELF) and electron orbits of $\mathrm{C}_{48}$ (Fig.3). Seen from the PDOS (Fig.3a), the conductive electrons are almost from the atoms at $\mathrm{C} 3$ and $\mathrm{C} 4$ positions, which are corresponding to the $s p^{2}$ atoms marked as green and red ones, respectively. Other $s p^{3}$ atoms in structure have no contribution to the conductivity due to the saturation of chemical bonds. Because the $s p^{2}$ atoms are linearly linked each other along $z$ axis, it is easy to conclude their $z$-axis conductivity. However, the key is to understand the electron coupling between $s p^{2}$ layers in order to judge the electrical eonductivity behavior in $x-y$ plane. As is well-known, ELF is used to identify the electron correlation useful to provide the bonding information. The values of ELF can be ranged from 0.0 to 1.0 , where 1.0 is corresponding to perfect localization, and 0.5 is corresponding to perfect electron delocalization. To analyze the bonding state between the $s p^{2}$ interlayers in structure, we draw out the-electronic isosurface map of ELF=0.5 (Fig.3b). Clearly, there are nө electron density between the $s p^{2}$-interlayers, indicating the perfect electron delocalization. Clearly, there is a gap of electron localization between the $s p^{2}$ 
interlayers, indicating the insulated interlayers. In addition, this gap is independent on the choice of isosurface value of ELF.

To further demonstrate the delocalized $\pi$ electrons and elucidate the conduction behavior of the $s p^{2}$ carbon chains along $z$ axis, we have calculated the electron orbits of $\mathrm{C}_{48}$ originated from the bands across the Fermi level (Fig.3c and 3d). Clearly, the $\pi$ electrons attached in the $s p^{2}$ carbon chains are separated in $x-y$ plane (Fig. 3d) but linked each other along $z$ axis (left in Fig.3c). Therefore, the eight divided $s p^{2}$ regions of unit cell are not conductive in $x-y$ plane, but only linearly conductive along $z$ axis. Furthermore, our calculation also shows that the electron orbits of $s p^{3}$ carbon atoms are all localized and separated in $x-y$ plane (Fig.3d) and $z$ axis (right in Fig.3c), thereby leading to their insulation in 3D. In short, the analyses of PDOS, ELF, and electron orbits support the consistent result that the $\mathrm{C}_{48}$ is a $3 \mathrm{D}$ carbon with peculiar $1 \mathrm{D}$ multithreaded conductivity. In short, the $\mathrm{C}_{48}$ is a $3 \mathrm{D}$ carbon with peculiar $1 \mathrm{D}$ multithreaded conductivity. 

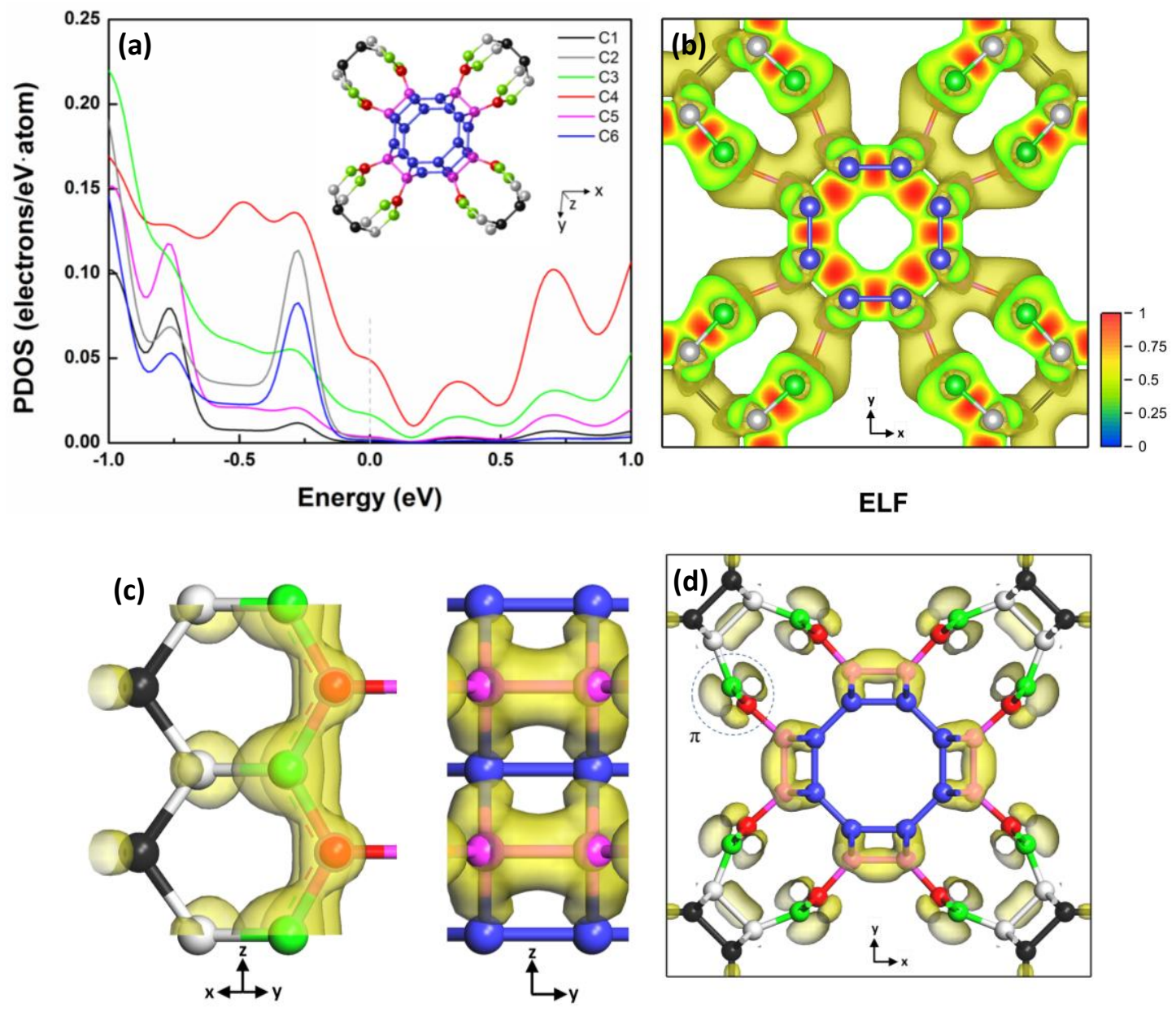

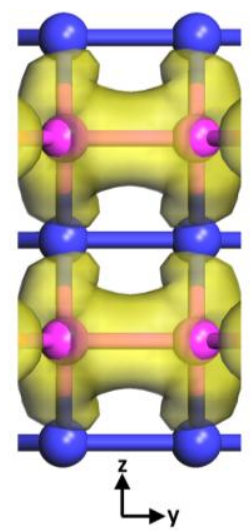

Electron orbits

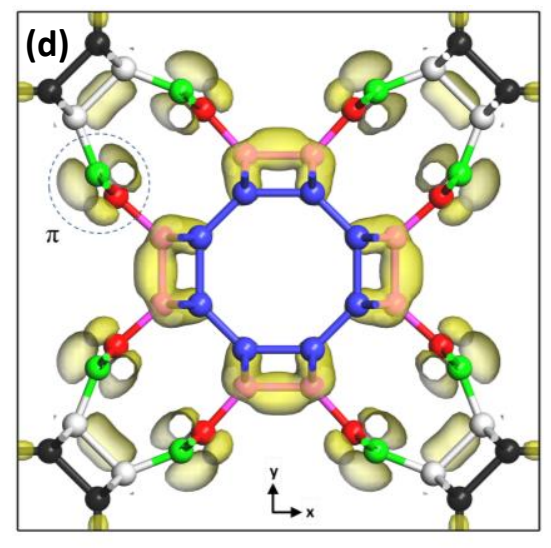

Electron orbits

Figure 3. (a) PDOS of $\mathrm{C}_{48}$. The inset is the crystal structure of $\mathrm{C}_{48}$ with six nonequivalent atomic positions marked by different colors corresponding to those in PDOS. Only the atoms located in $\mathrm{C} 3$ and $\mathrm{C} 4$ positions contribute to the conductivity. (b) ELF of $\mathrm{C}_{48}$ at isosurface equal to 0.5 representing the perfect electron delocalization. Elearly, there is no electron density between the $s p^{2}$ interlayers, resulting in the eight divided linear conductive paths composed of $\mathrm{C} 3$ and $\mathrm{C} 4$ atoms along $z$ axis. Clearly, there is a gap of electron localization between the $s p^{2}$ interlayers, indicating the interlayers are non-conductive. (c), (d) Electron orbits of $\mathrm{C}_{48}$ originated from the bands across the Fermi level. The selected isosurface is 0.0115 . The electron orbits are all insulted in $x-y$ plane (Fig. 3d), and the $\pi$ bonds attached in the $s p^{2}$ carbon chains are linked each other along $z$ axis, resulting in the linear conductivity (left in Fig. 3c). In comparison, the localized electron orbits of the $s p^{3}$ carbon atoms are still divided along $z$ axis, leading to their insulation in 3D (right in Fig. 3c).

The axial ( $z$ axis) and radial tensile strengths of $\mathrm{C}_{48}$ were further calculated. As shown in Fig.4, the tensile strengths along the [001], [100] and [110] directions are 
109.12 GPa with a strain of $0.25,45.36 \mathrm{GPa}$ with a strain of 0.22 , and $26.04 \mathrm{GPa}$ with a strain of 0.08 , respectively. The axial [001] tensile strength of $\mathrm{C}_{48}$ is about double that of current calculated $(12,12) \mathrm{SWCNTs}$, and is comparable with the in-plane intrinsic tensile strength of graphene reaching $130 \pm 10 \mathrm{GPa}$ [24]. Its radial tensile strength has been greatly improved, far higher than those of 1D carbon nanotubes and nanowires with weak Van der Waals force. The lowest tensile strength is along the [110] direction and the crystal would be cleaved along the [110] direction when the external stress exceeds $26.04 \mathrm{GPa}$. Notably, the re-enhancement along the [100] direction was observed during tension, and this is due to the successive bond destruction originated from $\mathrm{C} 2$ atoms and $\mathrm{C} 1$ atoms. In addition, the Vickers hardness is also estimated by Chen's hardness model[18, 19], and the $\mathrm{C}_{48}$ has a hardness of $24.26 \mathrm{GPa}$, implying that it is a hard material.

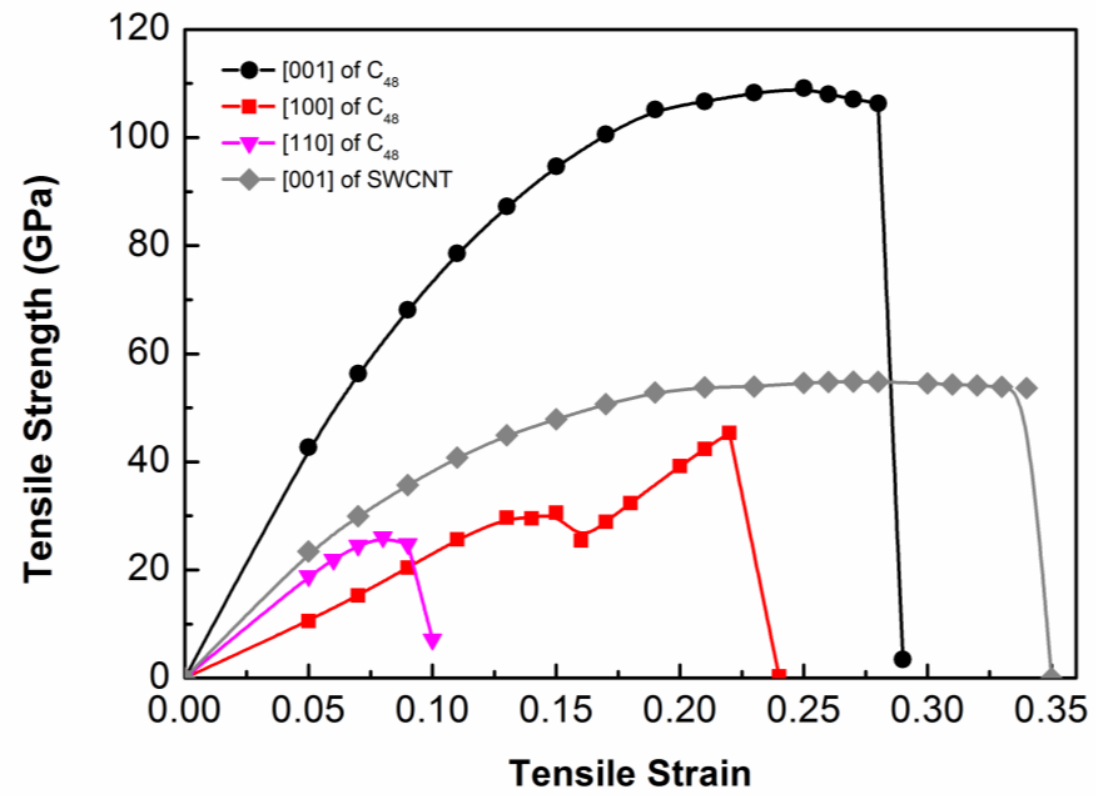


Figure 4. Calculated tensile strengths versus tensile strains of $\mathrm{C}_{48}$ along the [001], [100], and [110] orientations, respectively. In comparison, the strongest crystal orientation of $(12,12)$ SWCNTs is marked by [001].

We further provide the possible formation of $\mathrm{C}_{48}$ through compressing $(12,12)$ SWCNT bundle under hydrostatic pressure of $60 \mathrm{GPa}$ (Fig.5). Firstly, the rounded tubes in array were compressed into flat squares (Fig.5b). Further compression led to the formation of a screw-propeller-like polymer linked by the $s p^{3} \mathrm{C}-\mathrm{C}$ bonds at the corners (the green sticks, Fig.5c). Finally, the tubes were contracted and new $s p^{3}$ bonds at inner tube were formed to construct the crystal structure of $\mathrm{C}_{48}$ (Fig.5d). Recently, the growth of SWCNTs with specific chirality has attracted lots of research groups' attention, and now. The production of $(12,12)$ SWCNTs has been reported before, but the product has a low purity and also includes other chiral nanotubes[25-27]. Now high-yield production of $(12,6)$ SWCNTs with single chirality has been successfully achieved[28]. The large-scale synthesis of other high-purity SWCNTs with controllable chiralities is highly anticipated in near future, and then the predicted SWCNTs polymers including $\mathrm{C}_{48}$ may be easily synthesized. 


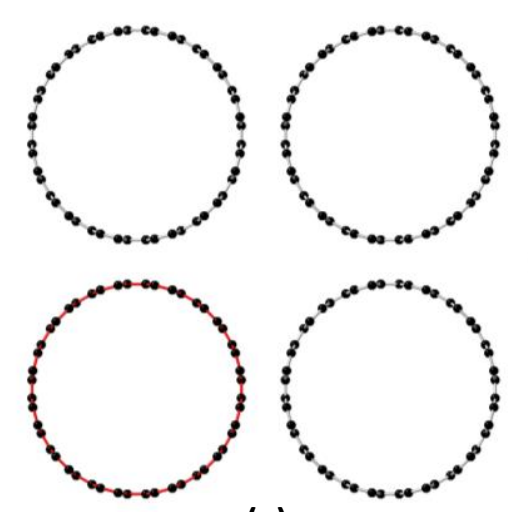

(a)

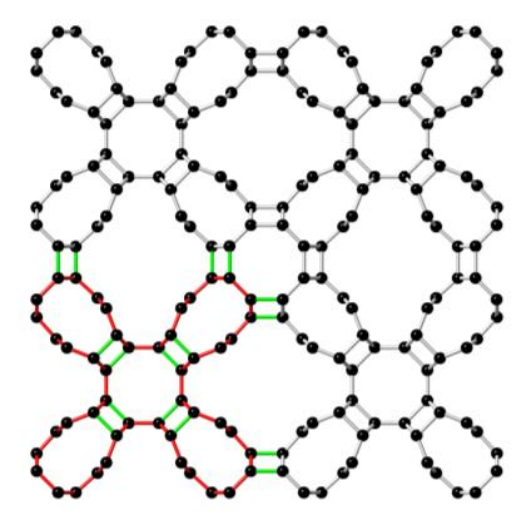

(d)

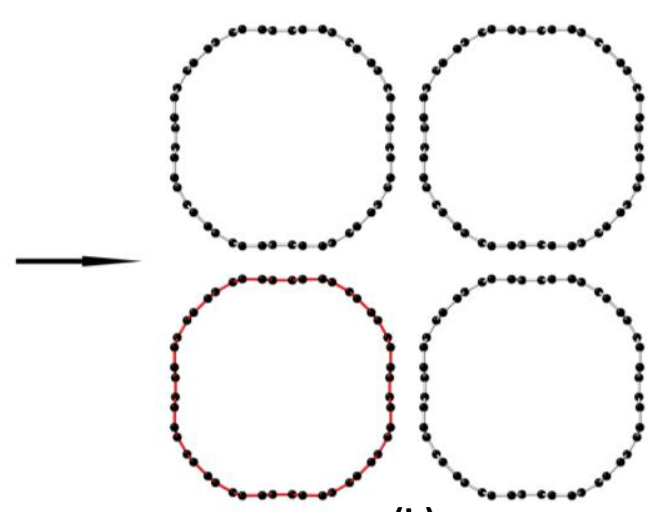

(b)

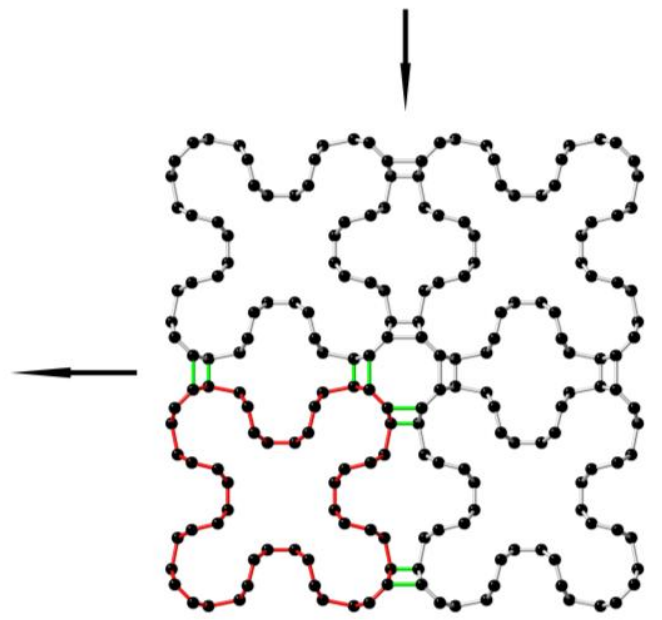

(c)

Figure 5. Formation of $3 \mathrm{D} \mathrm{C}_{48}$ by compressing $1 \mathrm{D}(12,12) \mathrm{SWCNTs}$ under hydrostatic pressure of $60 \mathrm{GPa}$. (a) Original nanotube array. During compression, the nanotubes are squashed into race track-like shape (b). Further compression induces the intertube (c) and intratube (d) bonding, and finally to form $\mathrm{C}_{48}$ (d). The green sticks represent the new $s p^{3}$ bonds during compression, and the region including red lines represents the unit cell of $\mathrm{C}_{48}$.

\section{Conclusions}

In sum, a novel $s p^{2}-s p^{3}$ hybridized carbon, i.e. $\mathrm{C}_{48}$, with a fascinating lilac-like structure has been proved to be mechanically and dynamically stable, and would be formed during compressing the large-diameter SWCNTs. Structurally, $\mathrm{C}_{48}$ has a tetragonal unit cell containing a $s p^{3}$ cage-like center surrounded by four $s p^{2}-s p^{3}$ petal-like tube configurations. The electron analysis shows that $\mathrm{C}_{48}$ has eight separated atom-scaled linear conductive channels in the unit cell, making it having 1D multithreaded 
conduction in 3D. This conducting behavior is distinct from the 1D conduction of 1D SWCNTs, 2D conduction of 2D graphene, and also 3D conduction of 3D graphite, which is unique in carbon materials. The 1D multithreaded conductive channels can allow straight and adjustable electron transports, and the 3D $s p^{2}-s p^{3}$ framework would support the excellent mechanical properties such as strength, moduli, and hardness, making $\mathrm{C}_{48}$ find potential application in various electronic nanodevices like field emission devices.

\section{Acknowledgements}

This work was supported by the National Science Foundation of China (Grants Nos. 51672238, 51421091, 51525205, 51332005, and 51272227), the National Basic Research Program of China (Grant No. 2011CB808205), and the Natural Science Foundation for Distinguished Young Scholars of Hebei Province of China (Grant No. E2014203150).

\section{References}

[1] Q. Li, Y. Ma, A.R. Oganov, H. Wang, H. Wang, Y. Xu, et al. Superhard Monoclinic Polymorph of Carbon, Phys Rev Lett 2009;102(17):175506.

[2] X.-F. Zhou, G.-R. Qian, X. Dong, L. Zhang, Y. Tian, H.-T. Wang, Ab initio study of the formation of transparent carbon under pressure, Phys Rev B 2010;82(13):134126.

[3] K. Umemoto, R.M. Wentzcovitch, S. Saito, T. Miyake, Body-Centered Tetragonal C 4 : A Viable sp ${ }^{3}$ Carbon Allotrope, Phys Rev Lett 2010;104(12):125504.

[4] J.T. Wang, C. Chen, Y. Kawazoe, Low-temperature phase transformation from graphite to $\mathrm{sp}^{3}$ orthorhombic carbon, Phys Rev Lett 2011;106(7):075501.

[5] Z. Zhao, B. Xu, X.F. Zhou, L.M. Wang, B. Wen, J. He, et al. Novel superhard carbon: C-centered orthorhombic $\mathrm{C}_{8}$, Phys Rev Lett 2011;107(21):215502.

[6] M. Hu, X. Dong, Y. Pan, B. Xu, D. Yu, J. He, A metallic carbon consisting of helical carbon triangle chains, J Phys Condens Matter 2014;26(23):235402. 
[7] M. Itoh, M. Kotani, H. Naito, T. Sunada, Y. Kawazoe, T. Adschiri, New metallic carbon crystal, Phys Rev Lett 2009;102(5):055703.

[8] S. Zhang, Q. Wang, X. Chen, P. Jena, Stable three-dimensional metallic carbon with interlocking hexagons, Proc Natl Acad Sci USA 2013;110(47):18809-13.

[9] M. Hu, Z. Zhao, F. Tian, A.R. Oganov, Q. Wang, M. Xiong, et al. Compressed carbon nanotubes: a family of new multifunctional carbon allotropes, Sci Rep 2013;3:1331.

[10] P.A. Schultz, K. Leung, E.B. Stechel, Small rings and amorphous tetrahedral carbon, Phys Rev B 1999;59(2):733-741.

[11] Z. Zhisheng, X. Bo, W. Li-Min, Z. Xiang-Feng, H. Julong, L. Zhongyuan, et al. Three dimensional carbon nanotube polymers, Acs Nano 2011;5(9):7226-34.

[12] T. Yildirim, O. Gülseren, Ç. Kılıı̧, S. Ciraci, Pressure-induced interlinking of carbon nanotubes, Phys Rev B 2000;62(19):12648-12651.

[13] Z. Zhao, X.-F. Zhou, M. Hu, D. Yu, J. He, H.-T. Wang, et al. High-pressure behaviors of carbon nanotubes, Journal of Superhard Materials 2012;34(6):371-385.

[14] Materials Studio, Version 6.0; Accelrys Inc.: San Diego, CA, 2011

[15] D.M. Ceperley, B.J. Alder, Ground State of the Electron Gas by a Stochastic Method, Phys Rev Lett 1980;45(7):566--569.

[16] J.P. Perdew, A. Zunger, Self-interaction correction to density-functional approximations for many-electron systems, Phys Rev B 1981;23(10):5048-5079.

[17] H.J. Monkhorst, J.D. Pack, Special points for Brillouin-zone integrations, Phys Rev B 1976;13(12):5188-5192.

[18] X.Q. Chen, H. Niu, D. Li, Y. Li, Modeling hardness of polycrystalline materials and bulk metallic glasses, Intermetallics 2011;19(9):1275-1281.

[19] X.Q. Chen, H. Niu, C. Franchini, D. Li, Y. Li, Hardness of T-carbon: Density functional theory calculations, Phys Rev B 2011;84(84):2669-2674.

[20] Z.-j. Wu, E.-j. Zhao, H.-p. Xiang, X.-f. Hao, X.-j. Liu, J. Meng, Crystal structures and elastic properties of superhard $\operatorname{IrN}_{2}$ and $\operatorname{IrN}_{3}$ from first principles, Phys Rev B 2007;76(5):054115.

[21] S. Pugh, Relations between the elastic moduli and the plastic properties of polycrystalline pure metals, Philos Mag 1954;45(367):823-843.

[22] X. Guo, L.-M. Wang, B. Xu, Z. Liu, D. Yu, J. He, et al. Unbinding force of chemical bonds and tensile strength in strong crystals, J Phys Condens Matter 2009;21(48):485405.

[23] A. Simon, Superconductivity and Chemistry, Angewandte Chemie International Edition in English 1997;36(17):1788-1806.

[24] C. Lee, X. Wei, J.W. Kysar, J. Hone, Measurement of the elastic properties and intrinsic strength of monolayer graphene, Science 2008;321(5887):385-388.

[25] R.M. Sundaram, K.K. Koziol, A.H. Windle, Continuous direct spinning of fibers of single-walled carbon nanotubes with metallic chirality, Adv Mater 2011;23(43):5064-5068.

[26] A. Mueller, K.Y. Amsharov, Synthesis of Robust Precursors for the Controlled Fabrication of $(6,6)$, $(8,8),(10,10)$, and $(12,12)$ Armchair Single-Walled Carbon Nanotubes, Eur J Org Chem

2015;(14):3053-3056.

[27] J.R. Sanchez-Valencia, T. Dienel, O. Gröning, I. Shorubalko, A. Mueller, M. Jansen, et al. Controlled synthesis of single-chirality carbon nanotubes, Nature 2014;512(7512):61-64.

[28] F. Yang, X. Wang, D. Zhang, J. Yang, D. Luo, Z. Xu, et al. Chirality-specific growth of single-walled carbon nanotubes on solid alloy catalysts, Nature 2014;510(7506):522-4. 

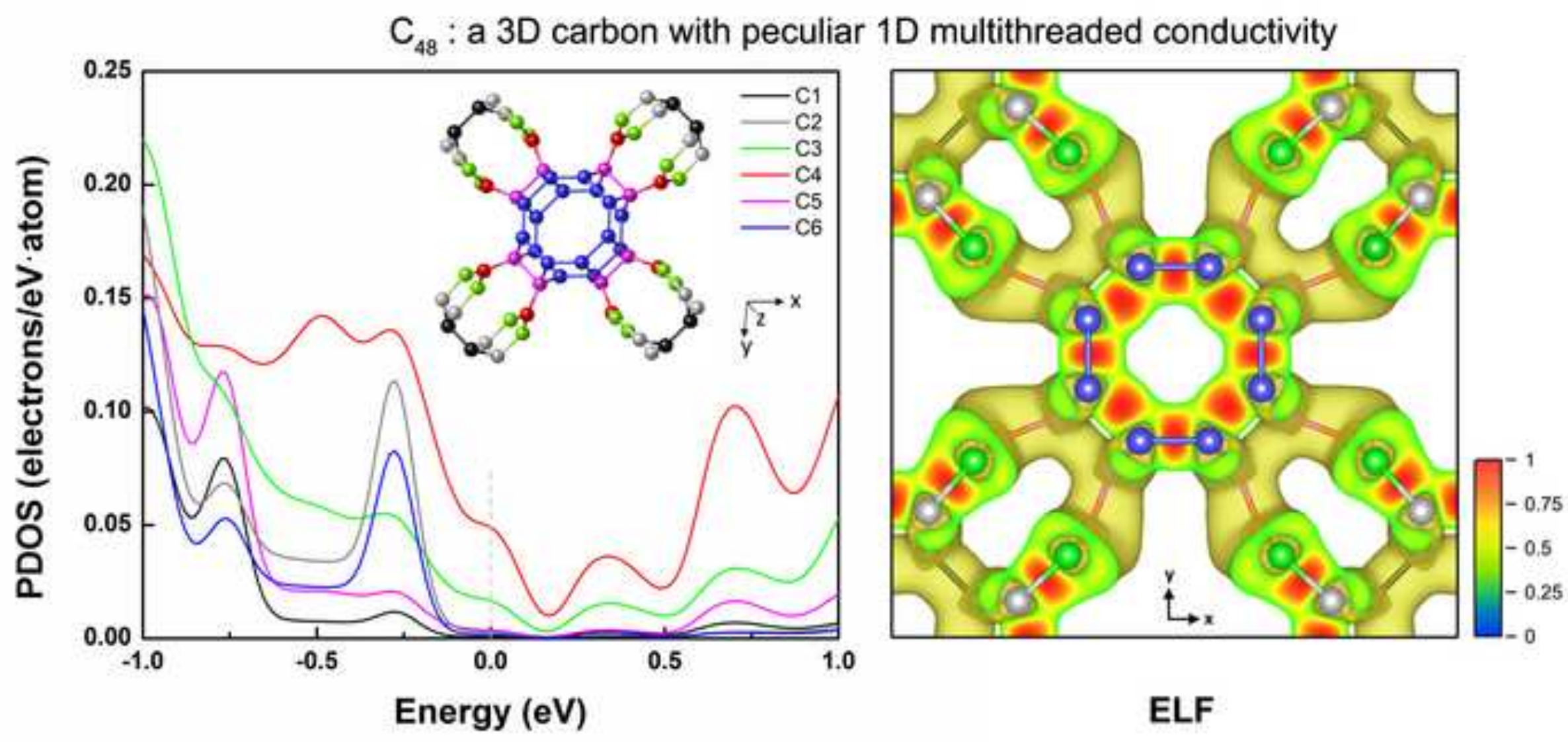